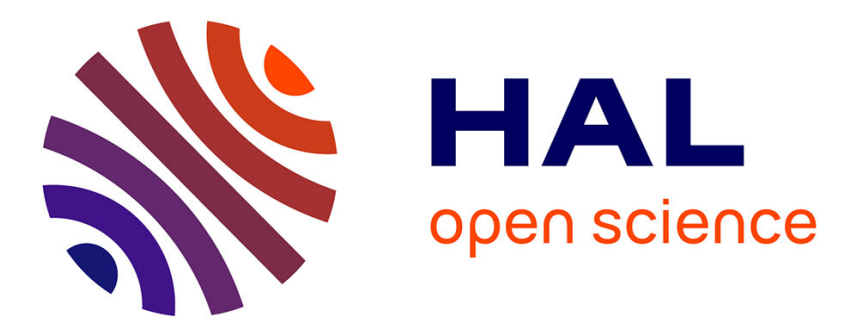

\title{
A Multiagent System to Model Human Action Based on the Concept of Affordance
}

Zoubida Afoutni, Rémy Courdier, Francois Guerrin

\section{To cite this version:}

Zoubida Afoutni, Rémy Courdier, Francois Guerrin. A Multiagent System to Model Human Action Based on the Concept of Affordance. 4th International Conference on Simulation and Modeling Methodologies, Technologies and Applications (SIMULTECH 2014), Aug 2014, Vienne, Austria. pp.644-651, 10.5220/0005141606440651 . hal-01466931

\section{HAL Id: hal-01466931 \\ https://hal.science/hal-01466931}

Submitted on 13 Feb 2017

HAL is a multi-disciplinary open access archive for the deposit and dissemination of scientific research documents, whether they are published or not. The documents may come from teaching and research institutions in France or abroad, or from public or private research centers.
L'archive ouverte pluridisciplinaire HAL, est destinée au dépôt et à la diffusion de documents scientifiques de niveau recherche, publiés ou non, émanant des établissements d'enseignement et de recherche français ou étrangers, des laboratoires publics ou privés. 


\title{
A Multiagent System To Model Human Action Based on the Concept of Affordance
}

\author{
Zoubida Afoutni ${ }^{1}$, Rémy Courdier ${ }^{1}$ and François Guerrin ${ }^{2}$ \\ ${ }^{1}$ Université de la Réunion, Laboratoire d'Informatique et de Mathématique,France \\ ${ }^{2}$ Centre de coopération internationale en recherche agronomique pour le développement (CIRAD), UR Recyclage et risque, \\ Montpellier, France \\ zafoutni@univ-reunion.fr, remy.courdier@univ-reunion.fr,francois.guerrin@cirad.fr
}

Keywords: Situated action, affordance, emergence, multiagent system.

\begin{abstract}
This paper presents a model to represent situated action in farming systems. The basic idea is to invert the classical vision of action that stipulates that action is a consequence of a decision process and a plan. We consider action as mainly dictated by a situation created by the interaction of the actor and his/her environment. Hence, action is a process that emerges from this situation. Thus, in this paper we treat the question of how to model action as an emergence process from the situations created by actors and their environment? For this, our model is based on multiagent system as well as on the affordance, emergence and stigmergy concepts (this paper emphasizes mainly the first two).
\end{abstract}

\section{INTRODUCTION}

Human action is the driving force of many systems, such as farming systems. Understanding these systems, namely with the aim of providing management support, requires a representation of human action as close to reality as possible. Thus we propose here a model to represent human action, taking into account explicitly its spatial and temporal dimensions. Our conception of human action is based on the theory of situated action (Suchman, 1987) which assumes that a realistic representation of action should not extract it from its occurrence situation nor reduce it to a pre-constructed plan. A defining feature of this theory is the concept that all resources required for action are located in the actor's environment. The latter is able to perform the appropriate action at any time, through the observation of his/her local environment. Thus it is the actor's environment that, in some way, dictates action, while the actor is performing it. This work is based precisely on the idea that the choice of the action to be performed, interrupted or cancelled is not the responsibility of the actor but rather of its environment. To implement our model we used a multiagent systems (MAS) modelling approach, along with the concepts of affordance, emergence and stigmergy. The first two concepts were used to represent action at an individual level. The concept of stigmergy is used to represent action at a collective level, specifically the indirect coordination between actors mediated by marks left in the environment. As this paper is focused on the representation of action at the individual level, therefore the coordination mechanisms will not be discussed (Afoutni, 2012; Afoutni et al., 2010). The choice of MAS was natural because it allows one to implement explicitly everything related to human activity and, as we will demonstrate, to situated action as well. Indeed, an action is performed by an actor embodied within a space. Its location limits his/her perception and therefore all actions that he/she can achieve. The MAS approach allows us to design our model using multiagent basic concepts: Agent, Environment and Interaction. Moreover, since we consider the actor's environment plays a crucial role in determining action in time and space, the environment must embody all the information needed for the actor to perform the appropriate actions. The concept of affordance (Gibson, 1986) is an elegant solution to implement our idea. Indeed, according to Gibson, an affordance represents a set of actions offered to an actor by the objects in his/her environment at a given time and location. This concept has been developed in various works to determine that affordance is rather an emergent property of the pair actor-environment (Stoffregen, 2003; Chemero, 2003). The concept of affordance is closely related to the notion of situation defined as a set of resources and constraints with which an actor interacts (Afoutni, 2012). 
Since the objects in an actor's environment are an integral part of the situation he/she encounters, defining a mechanism that could detect these affordances will lead to representing situated action. Wishing to answer the question: how can we detect affordances in the actor-environment system, we focused on the concept of emergence which is a promising way to model affordances. This paper is organized in three sections. The first section introduces briefly the concept of affordance followed by the relationship between affordance and emergence. The second section presents our model of representation of situated action. The third section is dedicated to the experimentation of the simulation model, and we conclude this work by a discussion.

\section{AFFORDANCE AND EMERGENCE}

The concept of affordance has its roots in the work of the psychologist Koffka (Koffka, 1935). However, Gibson improved this concept for his work on human direct visual perception (Gibson, 1986). The hypothesis of direct perception outlines that there is no need of a symbolic representation of the environment in the actor's mind nor an inference mechanism to extract the meaning of his/her percepts. Instead, any solicitation from the environment is perceived as an affordance directly by the actor. For example, a rigid horizontal surface affords an actor to walk; a chair affords a person to sit while it affords an ant to climb. However there is no consensus on "where is exactly the affordance in the pair actor-environment ", although there is unanimity that affordance is an emergent property of the actor-environment system (Luyat and Regia-Corte, 2009; Stoffregen, 2003). According to these authors, affordances exist only in the pair actor-environment, as the fruit of their interactions. This suggests that emergence can only be observed by an higher level than the actor-environment interaction's level. An affordance is thus not reducible to only one element of the actor-environment pair. We find similarities between this notion of affordance and the concept of emergence. The latter states that a system exhibits emergence when there are phenomena (properties, structures, behaviours, etc.) that arise from the interactions between the entities of the system at the "micro" level and become visible only at a "macro" (higher) level. Emerging phenomena are characterized by their novelty compared to the system's individual entities (Wolf and Holvoet, 2005).

Thus, since an affordance is a property originating from the pair actor-environment and since it is re- lated to an actor, the latter is capable of detecting this affordance and exploits it. Hence, the actor plays a double role. It is an element of the system leading to the emergence of the affordance as well as the observer who is able to discover this affordance through its knowledge on the system, as the work of (David and Courdier, 2009) suggests. These authors present the idea that experts are sometimes able to discover or observe an emergent phenomenon in some areas. This discovery would not have been possibly done by other people who do not have the necessary metaknowledge of the expert's domain. David et al. proposed to define emergence as a meta-knowledge and a methodology for detecting and reifying emerging phenomena on which we have based our work. Our model exploits all these concepts. However, according to our conception, an action is dictated by the environment and not by the actor. Thus we consider that the meta-knowledge which allows one to discover an affordance must be specified relative to the spatial environment.

\section{THE MODEL}

Our model is based on the idea that a multiagent system has an environment composed of a set of non-autonomous entities called environmentalentities, representing actors and passive entities of the studied system. The environmental-entities are located in a physical space in which they interact. This environment is controlled by a set of abstract agents called place-agents. Each place-agent is responsible for the entities operating in a well-defined area that we call place. The place-agent's role is:

- to observe within its perception field (its own place and neighbourhood) the environmentalentities and their interactions in order to detect and reify the possibly emerging affordances as well as to select and trigger the appropriate actions on the place it manages;

- to coordinate with other place-agents hence giving indirectly a consistent behaviour to the entire system.

This model therefore proposes to reverse the traditional view of MAS where the real-world actors are usually represented by "agents" (in the computerscience meaning) whereas the other system entities are represented as "objects of the environment". For us, the real-world actors are represented by nonautonomous entities the role of which is to perform the actions dictated by the place-agents. 
We present the model in four parts: environment, affordance, representation of action and agent architecture.

\subsection{Environment}

The environment is composed of environmentalentities as well as the physical space where they are located.

\subsubsection{Environmental Entities}

Environmental-entities represent the "actors" and all kind of passive objects of the studied system. An environmental-entity is characterized by a dynamic state and processes representing their internal dynamics. We distinguish two environmental-entity types: passive-object and actuator. A passive-object represents an entity that can only undergo an action. In the case of agricultural systems, a passive-object can be a stock of fertilizer, a plot, a tractor, etc. An actuator is an environmental-entity which possesses all mechanisms to implement an action but not the initiative capacity to undertake it which is the place-agents' role. Thus, an actuator may be a combination of an actuator with one or more passive-objects. For example, the action of ploughing can be done by the actuator "farmer-tractor-plough". If "farmer" is an actuator per se, the particular action of ploughing needs to combine it with "tractor" and "plough" otherwise considered as passive-objects. This environmentalentity characterization allows one to differentiate between entities that perform action from entities that undergo action. This helps answer a question related to action "who performs action?" The answer is the pair actor-environment likely to making affordances at some time and some place to emerge. Therefore, environmental-entities differ from agents by their lack of ability to take the initiative of acting (for actuators) and even to act (for passive objects).

Definition 1. An environmental-entity is a situated entity that carries information useful for the emergence of affordances. It is capable to execute or undergo an action, with internal processes that can modify its environment. It is characterized by the lack of autonomy and faculty of perception.

An environmental-entity e is characterized by:

- ide an unique identifier represented as an alphanumeric chain;

- $A_{e}$ a set of actions that it can perform or undergo: $A_{e}=\left\{a_{e_{1}}, a_{e_{2}}, \ldots, a_{e_{n}}\right\}$

- $E_{e}$ a set of variables that model its state: $E_{e}=\left\{e_{f_{1}}, e_{e_{2}}, \ldots, e_{e_{n}}\right\}$;
- De a set of functions that calculate the evolution of its internal state: $D_{e}: E_{e} \rightarrow 2^{E_{e}}$;

- If a set of functions that connect one of its state at a time $t$ with the list of actions it can perform or undergo: $\mathrm{I}_{f}: E_{e} \times t \rightarrow A_{e}$

- $p_{e}(t)$ a place where it is located at time $t$.

\subsubsection{Spatial Representation}

Representation of situated action necessarily involves the explicit representation of space. Thus, the physical space is where the environmental-entities are located. For example in agriculture, space is the set of land plots, buildings, equipment, roads, streams, etc. that compose the farm. The space in the model is a two dimensional Euclidean space. Each environmental entity is characterized by its position defined in a coordinate system. Space is regarded as continuous even if the environment is managed by place-agents. Each place-agent controls only a portion of space (its place) characterized by a vector of coordinates (see figure 1). Each place can have any geometric shape. Therefore the space composed by a set of places of equal sizes, with the same geometric shapes, is a discrete space (as mostly used in MAS); otherwise it is an irregular space as our model gives the modeller this possibility (see figure1).
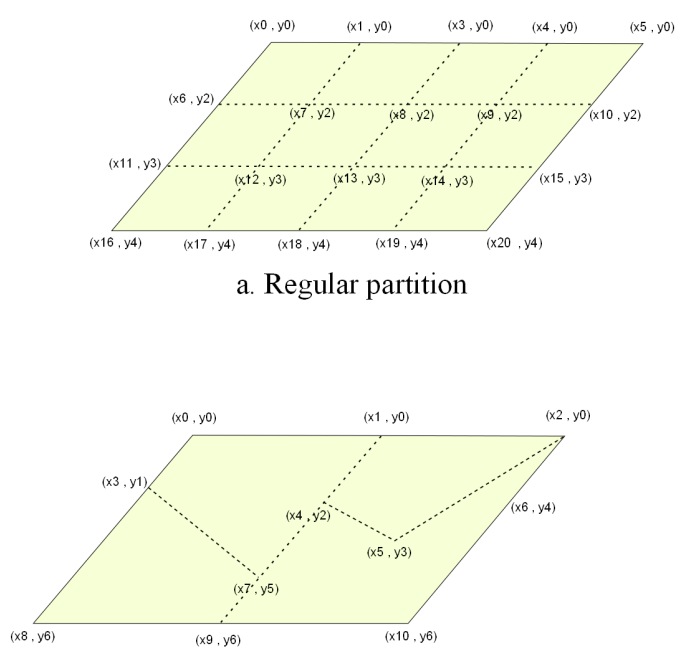

b. Irregular partition

Figure 1: Partition of continuous space into places 
Definition 2. A place $p$ is defined by:

- $i d_{p}$ an identifier of the place;

- $v_{p}$ a vector of coordinate points that specifies the geometry of $p$

- $i d_{g}$ an identifier of the agent managing the place $p$;

The space $P$ is a set made of the union of all elementary places $p_{i}$.

\subsection{The affordance Model}

Definition 3. An affordance is an emergent property, which can be detected at a macro level using metaknowledge held by agents-places that control the portions of the physical space (called "places") where environmental-entities are located.

Modelling affordances as emergent phenomena leads us to adapt the mechanisms involved in the detection and reification of emergent phenomena(David and Courdier, 2009).

\subsubsection{Detection of Affordance}

The detection of affordances by place-agents consists in observing at all time the states of all the environmental-entities located on the place they manage or their immediate neighbourhood making the list of actions that can be performed or dealt with by them. This observation allows place-agents to build a set of facts $K$ that represent the knowledge embedded in their environment and to discover affordances.

A fact is characterized by the 3-uple (environmental-entity, state/action).

After building $K$, we need to analyze this set to discover potential affordances. We use a set of functions $R_{\Phi}$ called affordance revealing functions. Every function's argument is the set $K$ of facts and its output is a set of potential affordances.

$$
R_{\Phi}=\left\{f: K^{n} \rightarrow \Phi\right\}, \quad n \in N
$$

Where $\Phi$ is the set of emerging affordances.

\subsubsection{Reification of Affordance}

The affordance detection phase outlines new affordances that will be used by the agent:

- to select one or more actions to perform;

- to improve the system with new knowledge that did not exist originally.

The second point is the key advantage of reification. Detected affordances will be reified into the environment through what we call a structure of affordance, characterized by: (i) the affordance (i.e. a possible action) (ii) the actuator(s)/passive object(s) causing the detected affordance.

Reification of affordances is subject to a set of conditions that specify whether an affordance can be reified and when it should be removed from the environment. Indeed, a structure of affordance may become invalid when the current state does not match the conditions that create this affordance.

Reification allows us to create knowledge in the system. The structures of affordances allow the agent to discover thereafter other affordances that could not have been detected with the basic entities (actuators and passive objects) of the system. The structures of affordances also mimic a memory embedded in the environment that can be used directly by an agent when the configuration of the environmental-entities located on its place has not changed between two simulation time steps.

\subsection{Representation of Action}

In this paper, we consider action as a noninstantaneous dynamic process, whose execution is constrained by the perceived conditions in the agent's environment. Thus, an action has a start date and end date and a duration. It may be interrupted or cancelled if the conditions necessary for its continuation no longer exist. An action may be in different states: running, interrupted, canceled.

Definition 4. An action is a dynamic process invoked by an agent, executed by an actuator, which can be undergo by a passive-object, characterized by a starting date, an ending date and a duration, conducted in a place of the space. It possesses a state and a priority.

\subsubsection{Dynamic State of an Action}

Our model is based on the representation formalism of action presented in (Guerrin, 2009). An action $a$ is represented by a binary function of time $(t)$, space $(P)$ an environmental-entity $(E)$ and constrained by a set of conditions $C_{a}(t, p, e)$ :

$$
S_{a}(t, p, e)=\left\{\begin{array}{ll}
1 & \text { if } \\
0 & \text { else }
\end{array} \quad C_{a}(t, p, e)\right.
$$

$p$ is the place where action is running, $t$ the current time, $e$ the set of environmental entities involved in the action. $\left.C_{a}(t, p, e)\right)$ is a logical proposition depending on time and space. When this proposition equals 1 , it means that the current situation allows the execution of action. Otherwise, three cases are possible: 
(i) the current state no longer allows the continuation of action (e.g. the current date value is greater than the end date), then the action will be definitively cancelled (ii) the current situation corresponds to the situation for the termination of an action, then the end date of action will be calculated (iii) the current situation requires the interruption of action. Thus, an action $a$ is represented as a dynamic process defined by a succession of time intervals.

\subsubsection{Action Temporal Bounds}

The starting and ending times $\left(t^{-}, t^{+}\right)$and the duration $\left(\tau_{a}(t)\right)$ of an action are also functions of time, determined by a condition $P_{a}^{ \pm}$.

$$
t_{a}^{ \pm}(t, p, e)= \begin{cases}t & \text { if } \quad P_{a}^{ \pm}(t, p, e) \\ t_{a}^{ \pm}\left(\max \left(0, t-\tau_{a}\right)\right) & \text { else }\end{cases}
$$

When the condition $P_{a}^{-}(t, p, e)\left(\operatorname{resp} P_{a}^{+}(t, p, e)\right)$ is satisfied it can trigger the corresponding action and calculate its start (or end) date, otherwise the value $t_{a}^{ \pm}$ is identical to its value at the previous time step.

\subsection{Agent Architecture}

Physical space and environmental entities are managed by a set of place-agents. Each of them is responsible for managing actions on its assigned place (figure 2). These actions are induced by the environmental-entities and other conditions observed on its place through detected affordances. Thus, the detection of affordances, the selection and update of the state of action as well as its place's state are the main duties of any place-agent.

\subsubsection{Perception}

When a place-agent perceives an environmentalentity, it may arise two cases:

- the environmental-entity $e$ perceived is on the place managed by the place-agent $g$;

- the environmental entity $e$ perceived is on the place managed by a neighbouring place-agent $g^{\prime}$.

In the first case, it could lead to trigger an action by the actuator bearing the capacity of performing it. In the second case, since a place-agent does not have the right to trigger an action with an actuator that is not on its own place, this could lead to initiate a behaviour of coordination with the neighbouring agent. Hence, we define two boolean perception functions: $\operatorname{Perc}_{e}$ and $\mathrm{Obs}_{e}$.
$\operatorname{Perc}_{e}(g, e)= \begin{cases}1 & \text { if } e \text { on place of } g \\ 0 & \text { else }\end{cases}$

$\operatorname{Obs}_{e}(g, e)= \begin{cases}1 & \text { if } e \text { on neighbouring place of } g \\ 0 & \text { else }\end{cases}$

Once the environmental-entities that are perceived by a place-agent are identified, the place-agent needs to collect facts potentially leading to the emergence of an affordance. A place-agent can thus perceive only the facts necessary to allow the emergence of affordances corresponding to the actions that could be performed on passive-objects or performed by actuators located on this place. The facts perceived by a placeagent should be restricted by a set of filters Filtre $_{K}$. These are functions that specify for each agent the facts at the environmental-entity's level that it can perceive. These filters are defined by:

$$
\text { Filter }_{k}: G \times E \rightarrow K
$$

\subsubsection{Detection of Affordances}

The perception phase allows a place-agent to acquire information on its local environment which represents all the facts. These facts may concern the information collected at environmental entities' level or about the general state of the environment. The facts from the environmental entities will allow the agent to help affordances to emerge using the affordance revealing functions $R_{\phi}$.

\subsubsection{Selecting an Action}

Once the list of affordances has been built, the placeagent needs to select one or more actions which can run concurrently. This selection is based on both preconditions necessary for triggering actions $P_{a}^{ \pm}$and the priorities assigned to actions.

When priorities are assigned a priori they are fixed and do not change during the simulation. But they could be dynamically assigned based on certain time-varying parameters. For example, some actions in the agricultural system cannot be performed outside of their earliest and latest completion dates. These actions become urgent when their end dates at the latest approach. The priority of an action is known using a function Priorite $_{a}$ that returns an integer.

The end of the selection phase of action is marked by the calculation of its starting date, using the equation (3). 


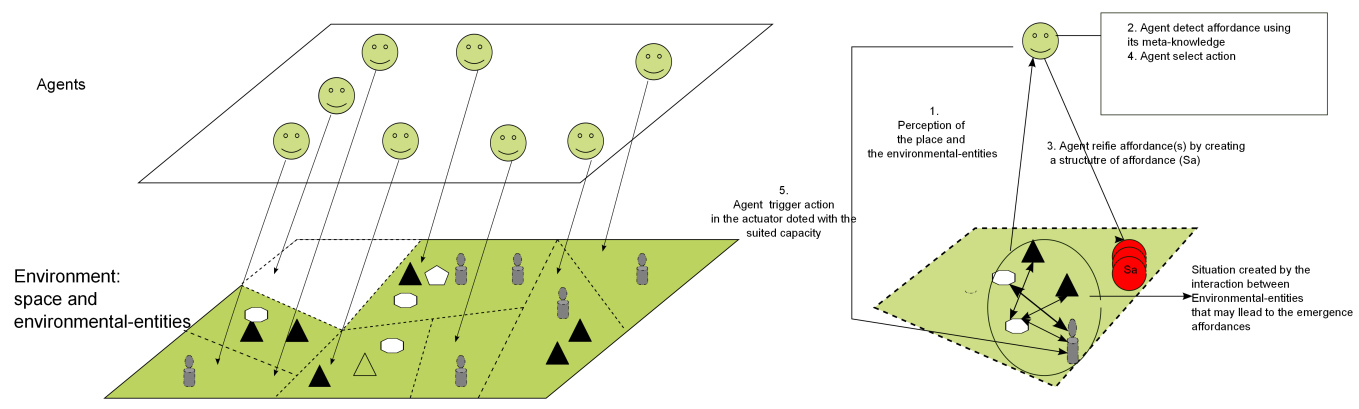

Figure 2: The relationship between the layer of agent and the environment

\subsubsection{Triggering an Action}

After selecting an action and calculating its starting date, the action will be triggered at the concerned actuator's level. Concretely triggering the action consists in bringing to ' 1 ' its state and in triggering processes implemented at the environmental-entities' level representing the effects of this action on the environment.

\subsubsection{Checking During an Action}

Given that action depends on a situation, its course is influenced by the evolution of this situation. Thus, the place-agent may be required to suspend, cancel or terminate the current action. This phase consists in checking at the beginning of each simulation cycle if the conditions of action's execution are still valid. If they are no longer valid, there are two cases:

- the place-agent interrupts its action;

- the place-agent notes that the conditions for completion of action $P_{a}^{+}(t, p, e)$ are valid. It calculates the ending date using equation ( 3 ) and informs the environmental-entity concerned of the end of action.

\section{CASE STUDY}

\subsection{Implementation}

A prototype of the proposed model has been implemented using the simulation platform AnyLogic (AnyLogic, 2010). AnyLogic can simulate models based on multiple modelling approaches: discrete event simulation as well as systems dynamics and multi-agent systems. It is based on the object-oriented language Java. Regarding the multi-agent approach, AnyLogic offers two types of entities: Agent and ActiveObject. ActiveObject can be understood as objects in object-oriented programming. The behaviour of objects (i.e. any entity of the model) can be implemented using state charts, simple functions, data flow diagrams, activity diagrams, etc.

\subsection{Description of the Simulated System}

Figure 3 gives an overview of the simulation of a model based on a GIS map with two farms. Farm 1 is made of 4 plots and 1 livestock building. Farm 2 is made of 3 plots.

The simulation aim is to represent the actions executed at the farm level concerning the livestock (e.g. emptying manure stocks) and crop cultivation of carrots and potatoes (e.g. ploughing, fertilization, etc.).

\subsection{Modelling of the Simulated System}

\subsubsection{Environment}

The environment is made by the physical 2-D space as well as all environmental-entities. In this model implementation, the physical space is actually partitioned into regular square-shaped cells although it is considered as continuous. Passive-objects are the stocks of manure, of fertilizers, the crop plots, etc. Actuators are the entities that can perform actions such as the farmer, the farmer and his tractor equipped with plough or other tools, etc.

\subsubsection{Agents}

We have three types of agents: plot-agent, buildingagent and road-agent. The distinction between these 


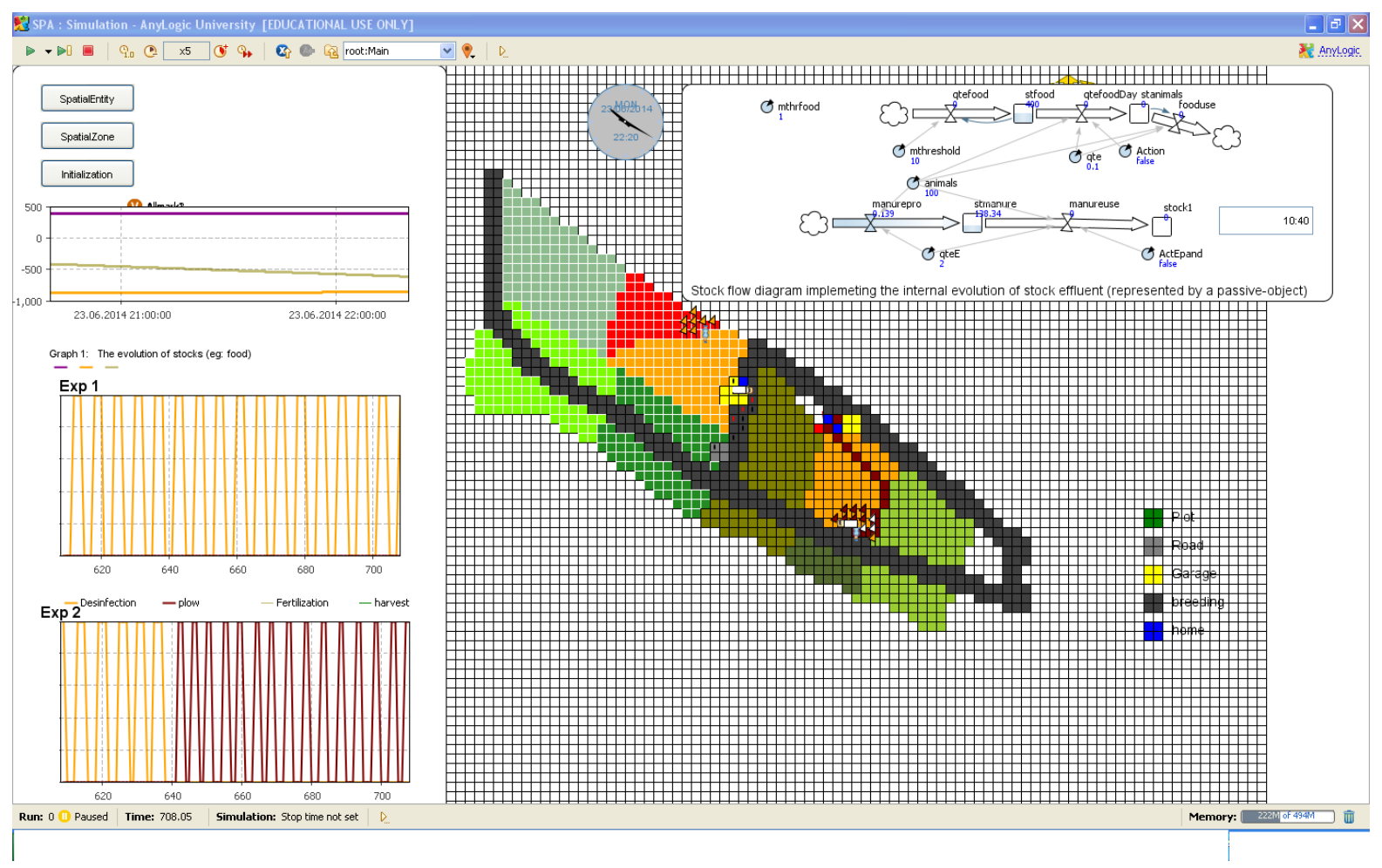

Figure 3: Simulation interface of the prototype implementing our action conceptual model using the AnyLogic platform.

three types is based on the actions that can be performed on the place controlled by every type of agents. For example, plot-agents have the ability to "fertilize the soil" but not to "empty the manure stock" (in fact to order relevant actuators to do that for them). But the latter action can be ordered by the livestock-building-agent. Thus, the type of agent constrains its perceptions and therefore all the facts $K$ that can be constructed at each simulation time step.

The affordance revealing and reification functions are based on the type of agent. Consequently, emerging affordances on a place $p$ correspond to the actions that can be performed on $p$. Each agent is assigned a place to manage, as shown in figure 3 on the cell grid representing space in this example.

\subsection{Output}

The graph 1 in figure 3 shows the evolution of the manure and livestock feed stocks that are located at the places managed by the livestock-building-agent. The behaviour of these two entities was implemented using the data flow diagrams shown on figure 3 .

Graph 2 shows the actions running in farm 1 (soil disinfection).

Graph 3 shows the actions running in Farm 2 (ploughing).
These graphs illustrate the actions performed in the agricultural system simulated. They show that the implemented MAS based on our conceptual model can simulate in a consistent manner a set of two farming systems without using any planning process. Although the capability of the model to account for the coordination of actions (namely implicit coordination based on stigmergy) has not yet been demonstrated here, this first result is a preliminary proof-of-concept of our model.

\section{DISCUSSION}

Our paper focuses on situated multi-agent systems (Weyns et al., 2007). One key-aspect that characterizes situated MAS is the role of the environment. Several functions are assigned to it: structuring entities within the system, communication and coordination between agents, etc.

We have shown that using the concepts of affordance and emergence allows us to create a 'smarter' environment for the agents using the knowledge carried by actuators and passive objects. The affordance detection mechanisms allow emerging actions of agents to be performed in time and space. Reification of these affordances as 'structures of affor- 
dances' provides the agents with a memory of past affordances. This avoids agents to recalculate their affordances at the next simulation step when nothing has changed in the meanwhile. Thus, it allows the modeller to add in the simulation scenarios as many actions as necessary without affecting the design of agents and the environment represented in the model. Indeed, we only have to inject new affordance revealing and reification functions to modify the simulation scenarios. Therefore a modification of the studied system does not imply its global destabilization nor restarting its whole design.

One model feature lies in the fact that the decision of the action to be performed is attributed to the environment through the concept of place-agents. Each place-agent manages a part of the physical space specified by a place where environmental-entities may be located be they mobile or static. This is in perfect harmony with the theory of situated action which states that actions are mainly determined at each instant by the situation in which the actor is. Hence, no overall strategy or central plan is necessary. Several other advantages can be dragged out from such a representation: the ability to simulate complex activity systems, to process both parallel and sequential actions and interactions, to account for interruption and resumption of actions, delays, etc.

Our prototype allowed us todate to validate the proposed concepts on a simple case study. To make it more useful to agronomists, the next step is to validate the model on more realistic complex situations. Moreover, the proposed conceptual model has been designed on a level of abstraction independent of the field of agricultural applications. We also plan to test our approach in other areas involving human actions.

\section{ACKNOWLEDGEMENTS}

The authors thank Dr. Yassine Gangat that helped significantly to improve the English text of this article.
Chemero, A. (2003). An outline of a theory of affordances. Ecological Psychology, 15(2):181-195.

David, D. and Courdier, R. (2009). See emergence as a metaknowledge - A way to reify emergent phenomena in multiagent simulations? In International Conference on Agents and Artificial Intelligence, pages 564569.

Gibson, J. (1986). The Ecological Approach to Visual Perception, Resources for Ecological Psychology. Lawrence Erlbaum and Associates, New Jersey.

Guerrin, F. (2009). Dynamic simulation of action at operations level. Autonomous Agents and Multiagents Systems, 18(1):156-185.

Koffka, K. (1935). Principles of Gestalt psychology. Harcourt, Brace and Company, NewYork.

Luyat, M. and Regia-Corte, T. (2009). Les affordances: de James Jerome Gibson aux formalisations récentes du concept. Année psychologique, 109(2):297-332.

Stoffregen, T. (2003). Affordances as properties of the animal environment system. Ecological Psychology, 15(2):115-134.

Suchman, L. (1987). Plans and situated actions: The problem of human-machine communication. Cambridge University Press, Cambridge, MA, USA.

Weyns, D., Omicini, A., and Odell, J. (2007). Environment as a first class abstraction in multiagent systems. $A u$ tonomous Agents and Multi-Agent Systems, 14(1):530.

Wolf, T. D. and Holvoet, T. (2005). Emergence versus self-organisation: Different concepts but promising when combined. Engineering self-organising systems, pages $1-15$.

\section{REFERENCES}

Afoutni, Z. (2012). Modelling situated action based on affordances and stigmergy. In IEEE Sixth International Conference on Self-Adaptive and Self-Organizing Systems, pages 175-180.

Afoutni, Z., Martin-Clouaire, R., Courdier, R., and Guerrin, F. (2010). Coordination of activities: application of some concepts and formalizations to agricultural systems simulation. In International Conference on Modeling and Applied Simulation, pages 25-31.

AnyLogic (2010). http://www.anylogic.com. 